\title{
Detecção de Fungos em Sementes de Acca sellowiana (Berg) Burret
}

\author{
Vinícius Spolaor Fantinel ${ }^{1}$, Luciana Magda de Oliveira ${ }^{1}$, Ricardo Trezzi Casa ${ }^{1}$, \\ Priscilla Félix Schneider ${ }^{1}$, Emerson Couto da Rocha ${ }^{1}$, Dalciana Vicente ${ }^{1}$, \\ Marluci Pozzan ${ }^{1}$ \\ ${ }^{1}$ Universidade do Estado de Santa Catarina - UDESC, Lages/SC, Brasil
}

\begin{abstract}
RESUMO
Acca sellowiana é uma espécie frutífera nativa da Região Sul do Brasil que está despertando grande interesse econômico devido ao alto potencial organoléptico de seus frutos. A principal forma de propagação da espécie é via sexuada. Objetivando determinar a qualidade sanitária das sementes, foram comparadas três formas de deteç̧ão de fungos, com ou sem a assepsia superficial, em sementes oriundas de quatro municípios do Estado do Rio Grande do Sul e de três municípios do Estado de Santa Catarina. Foram testados os meios de cultura Batata-Dextrose-Ágar (BDA), V8 (suco de tomate) e o método Blotter Test. Foram identificados os seguintes fungos: Aspergillus niger, Aspergillus flavus, Penicillium sp., Colletotrichum gloeosporioides, Fusarium sp., Curvularia sp., Alternaria sp., Trichoderma sp., Epicoccum sp. e Phomopsis sp. Os meios agarizados são mais sensíveis na detecção de fungos em sementes de goiaba serrana. A assepsia das sementes reduz a incidência de fungos infestantes.
\end{abstract}

Palavras-chave: goiaba serrana, patologia de sementes, semente florestal.

\section{Detection of Fungi in Acca Sellowiana (Berg) Burret Seeds}

\begin{abstract}
Acca sllowiana (Berg) Burret is a native fruit species in southern Brazil, which is attracting great interest due to the high economic potential of its fruit organoleptic potential; the main form of propagation of the species is sexual way. Aiming to determine the sanitary quality of seeds, three forms of detection of fungi were compared with and without surface sterilization. The seeds were derived from four counties in the state of Rio Grande do Sul and three counties in the state of Santa Catarina. The culture media of Potato Dextrose Agar (PDA), V8 (tomato juice) and the method of "Blotter Test" were tested. The following fungi were identified: Aspergillus niger, Aspergillus flavus, Penicillium sp., Colletotrichum gloeosporioides, Fusarium sp., Curvularia sp., Alternaria sp. Trichoderma sp. Epicoccum spp. and Phomopsis sp. The agarized media are more sensitive in the detection of fungi in Acca sellowiana seeds. The sterilization of seeds reduces the incidence of weed fungi.
\end{abstract}

Keywords: Feijoa, seed pathology, forest seed. 


\section{INTRODUÇÃO}

Acca sellowiana (Berg) Burret, popularmente conhecida por goiaba serrana, goiaba da serra ou feijoa, pertence à família Myrtaceae, que compreende cerca de 130 gêneros e aproximadamente 4 mil espécies descritas (Souza \& Lorenzi, 2005). É uma espécie arbórea nativa da América do Sul, e, no Brasil, ocorre desde o Paraná até o norte do Rio Grande do Sul. A espécie mostra-se adaptada a condições de clima frio, ocorrendo com maior frequência em áreas com altitude superior a 800 m (Amarante \& Santos, 2011).

No Brasil, estudos de mercado desenvolvidos em dois centros comerciais do Estado de Santa Catarina (Florianópolis e Blumenau) também demonstraram a existência de um mercado promissor, sendo que a estimativa média de comercialização dos frutos foi de aproximadamente $\mathrm{R} \$ 5,00 \mathrm{~kg}^{-1}$ (Santos et al., 2011), demonstrando que embora ainda não exista uso comercial expressivo da fruta no país, existe potencial para tanto.

A principal forma de propagação da goiaba serrana é por semente, podendo ainda propagar-se por estaquia, enxertia e micropropagação. A cultura da goiaba serrana pode ser afetada pela ocorrência de doenças causadas por fungos que têm seus agentes causais transmitidos por sementes, como é o caso de Colletotrichum gloeosporioides (Penz.) Sacc., agente causal da antracnose em frutíferas. Este fungo é cosmopolita, causa podridão de raízes, podridão e manchas negras nos frutos, dessecamento dos ramos, redução na germinação de sementes e mortalidade de plântulas (Sutton, 1992).

O estabelecimento do Banco Ativo de Germoplasma (BAG) da espécie, no Brasil, se deu inicialmente na Estação Experimental de Videira, SC, o qual chegou a ser composto por 160 acessos, com três plantas por acesso (Ducroquet, 1996). Contudo, devido à alta incidência do fungo C. gloeosporioides constatada já em 1990, a Empresa de Pesquisa Agropecuária e Extensão Rural de Santa Catarina (Epagri) transferiu o BAG para a estação experimental de São Joaquim, na qual tinha sido verificada menor incidência da doença devido às condições climáticas menos favoráveis ao desenvolvimento do fungo. Atualmente conta-se com cerca de 300 acessos, a maioria procedente do Estado de Santa Catarina, além de exemplares do exterior.
Com o advento do livre comércio, muitos países estão redefinindo suas exigências fitossanitárias, com o objetivo de prevenir a introdução de patógenos devastadores em seu território. Independentemente da metodologia de detecção, a especificidade, a sensibilidade, a confiabilidade, a eficiência do ensaio e uma compreensão de tolerância de patógenos em um lote de sementes precisam ser consideradas antes de uma técnica ser aceita como teste clínico sanitário de semente. Quando aceitáveis, os testes sanitários são ferramentas úteis para a gestão de risco de doença e rotineiramente utilizados na avaliação da qualidade das sementes (Maddox, 1998), além disso, há de se considerar, também, a necessidade do estabelecimento de padrões sanitários para sementes de espécies florestais no Brasil.

Entre os protocolos indicados pelo Ministério da Agricultura, Pecuária e Abastecimento - MAPA (Brasil, 2009) está a inspeção visual das amostras de sementes, exame da suspensão de lavagem de sementes, meio ágar sólido (Batata-Dextrose-Ágar ou MEA), método do papel de filtro e específicos para determinadas espécies fúngicas.

Têm sido publicados diversos trabalhos relacionados à detecção de fungos em sementes de diferentes culturas, sendo o teste com papel de filtro Blotter Test o mais conhecido e utilizado, embora a incidência de infestantes como fungos e bactérias possa impedir a frutificação dos fungos-alvo, dificultando a sua identificação e quantificação, sobretudo dos de crescimento lento (Reis \& Almeida, 1999).

Reis \& Casa (2007) descrevem outras formas de detecção de fungos, com possibilidade de uso em análises rotineiras de patógenos de sementes, como os meios seletivos e semisseletivos. Os meios de cultura devem ser utilizados quando outros não ofereçam condições adequadas para crescimento vegetativo, esporulação e detecção de fungos que produzam colônias características (Lucca, 1987). Os meios de cultura favorecem a germinação das sementes, podendo prejudicar a identificação dos fungos a elas associados (Coutinho et al., 2001). O meio ágar suco de tomate mais $6 \%$ de cloreto de sódio não favorece a germinação das sementes, facilitando a identificação de fungos por as sementes serem examinadas inteiras (Neergaard, 1983). 
O presente trabalho teve como objetivo comparar três formas de detecção de fungos (os meios de cultura BDA (Batata-Dextrose-Ágar), V8 (suco de tomate) e o método Blotter Test) em sementes de goiaba serrana oriundas dos estados do Rio Grande do Sul e Santa Catarina, submetidas ou não a assepsia superficial.

\section{MATERIAL E MÉTODOS}

\subsection{Procedências das sementes}

Para a realização do experimento foram utilizados sete lotes de sementes de goiaba serrana (Tabela 1). As sementes de Arroio do Tigre, Camaquã, Cachoeira do Sul e Venâncio Aires foram obtidas através do banco de sementes do Viveiro Florestal da Universidade Federal de Santa Maria (UFSM). As sementes dos lotes São Joaquim e Lages foram obtidas diretamente nas estações experimentais da Epagri, nos municípios de São Joaquim, SC, e Lages, SC (Tabela 1). Assim, as sementes desses seis lotes foram coletadas, processadas e armazenadas até o envio para a realização dos experimentos, pelo banco de sementes de onde foram adquiridas. Já para o lote Concórdia, após a colheita, as sementes foram extraídas por meio de maceração em água corrente, secas em temperatura ambiente por dois dias e armazenadas em câmara fria à temperatura de $10{ }^{\circ} \mathrm{C}$ e umidade relativa de $45 \%$ em embalagem de papel, por sete dias.

Quando adquiridas, as sementes estavam acondicionadas em embalagens de papel e identificadas com nome da espécie, local e data de coleta. Para a amostra CDIA, as sementes foram extraídas e beneficiadas no Laboratório de Sementes do CAV/UDESC, logo após passaram por uma secagem em temperatura ambiente, sendo posteriormente realizados os testes.
De acordo com a classificação climática de Köppen, os municípios do Estado do Rio Grande do Sul (AT, CMQ, CS, VA) e o município de Concórdia, situado no Estado de Santa Catarina, têm clima subtropical com verão quente (Cfa), com temperaturas superiores a $22^{\circ} \mathrm{C}$ no verão e com mais de $30 \mathrm{~mm}$ de chuva no mês mais seco. Já os municípios de Lages, SC, e São Joaquim, SC, caracterizam-se por apresentar inverno seco e verão ameno (Cfb), de acordo com a mesma classificação. A temperatura média do mês mais quente é inferior a $22^{\circ} \mathrm{C}$ (WREGE et al., 2011).

\subsection{Avaliação da sanidade das sementes}

Foram realizados três testes de sanidade em sementes: 1) Meio de cultura de BDA (extrato de $200 \mathrm{~g}$ de batata $+20 \mathrm{~g}$ de dextrose $+20 \mathrm{~g}$ de ágar/L); 2) Meio de cultura de V8 [200 $\mathrm{ml}$ de suco V8 (Tomato Juice) $+4,5 \mathrm{~g}$ de $\mathrm{CaCo}^{3}+17 \mathrm{~g}$ de ágar e $800 \mathrm{ml}$ de água destilada]; e 3) Método Blotter Test (Fernandez, 1993; Brasil, 2009).

a) Meio de cultura de batata-dextrose-ágar (BDA) e Meio de cultura de suco de tomate (V8): O meio de cultura de BDA consistiu de $200 \mathrm{~g}$ de batata fatiada cozidas em $500 \mathrm{~mL}$ de água destilada; em um erlenmeyer de $2.000 \mathrm{~mL}$ de capacidade foi adicionado o caldo da batata, $20 \mathrm{~g}$ de ágar e $20 \mathrm{~g}$ de dextrose, ajustando-se o volume final para $900 \mathrm{~mL}$, autoclavado a $120^{\circ} \mathrm{C}$ por 20 minutos. O meio V8 consistiu de $200 \mathrm{ml}$ de suco V8 (Tomato Juice), 4,5 g de $\mathrm{CaCO}_{3}, 17 \mathrm{~g}$ de ágar e $800 \mathrm{~mL}$ de água destilada, com posterior autoclavagem a $120^{\circ} \mathrm{C}$ por 20 minutos.

Após a autoclavagem, o substrato foi resfriado até atingir a temperatura de aproximadamente $45^{\circ} \mathrm{C}$. Em câmara de fluxo laminar, foram dissolvidos $0,2 \mathrm{~g}$ de sulfato de estreptomicina em $100 \mathrm{~mL}$ de água destilada

Tabela 1. Procedência e data de coleta de sementes de goiaba serrana.

Table 1. Origin and date of collection of seeds of guava feijoa.

\begin{tabular}{ccc}
\hline Amostra & Procedência & Data de coleta \\
\hline AT & Arroio do Tigre, RS & $07 / 03 / 2012$ \\
\hline CMQ & Camaquã, RS & $12 / 05 / 2012$ \\
CS & Cachoeira do Sul, RS & $30 / 03 / 2012$ \\
VA & Venâncio Aires, RS & $30 / 12 / 2011$ \\
CDIA & Concórdia, SC & $13 / 02 / 2013$ \\
LG & Lages, SC & $10 / 02 / 2013$ \\
\hline SJ & São Joaquim, SC & $15 / 12 / 2012$ \\
\hline
\end{tabular}


esterilizada, a qual foi adicionada aos meios BDA e V8 (Fernandez, 1993).

Cem sementes foram desinfestadas em hipoclorito de sódio a $1 \%$ por 30 segundos e álcool $70 \%$ antes do plaqueamento, depois foram lavadas três vezes em água destilada, para remover o resíduo do hipoclorito. Após a desinfestação, as sementes foram postas para secar sobre papel-filtro e, em seguida, plaqueadas em BDA e V8. Outras 100 sementes foram submetidas a testes de sanidade em meio agarizados sem passar por desinfestação, a fim de se compararem os resultados. Para os testes, foram utilizadas repetições de 25 sementes.

A incubação ocorreu em câmara de crescimento com temperatura $22{ }^{\circ} \mathrm{C} \pm 3{ }^{\circ} \mathrm{C}$, com 12 horas de fotoperíodo. A avaliação, para ambos os testes de sanidade, foi realizada observando-se as estruturas fúngicas, em microscópio estereoscópico e óptico, e a identificação dos fungos foi realizada com o auxílio da chave de identificação (Barnett \& Henter, 1998). Os dados da incidência dos fungos foram expressos em percentagem.

b) Blotter Test: foram utilizadas 100 sementes divididas em quatro repetições, desinfestadas com álcool $70 \%$, hipoclorito de sódio a $1 \%$ e em seguida lavadas com água destilada e 100 sementes sem desinfestação. As sementes foram distribuídas na proporção de 25 sementes por caixa de acrílico transparente (gerbox) forradas com duas folhas de papel filtro, as quais foram previamente esterilizadas em autoclave a $1 \mathrm{~atm}\left(120^{\circ} \mathrm{C}\right)$ por 20 minutos e umedecidas com água destilada esterilizada. A incubação foi realizada em câmara de crescimento com temperatura controlada a $22^{\circ} \mathrm{C} \pm 3^{\circ} \mathrm{C}$, fotoperíodo de 12 horas, durante sete dias, após os quais ocorreu a avaliação e identificação dos fungos.

\subsection{Procedimento estatístico}

O delineamento experimental utilizado foi o inteiramente casualizado, com quatro repetições para cada teste realizado. Para a análise de variância, os dados de percentagem foram transformados pela fórmula ARCSEN $[(\mathrm{x}+0,5) /(100)]^{1 / 2}$, pois não foi constatada normalidade dos mesmos. A comparação das médias foi feita pelo teste de Tukey a $5 \%$ de probabilidade e o programa utilizado para as análises estatísticas foi o SISVAR (Ferreira, 2000).

\section{RESULTADOS E DISCUSSÃO}

Foram identificados nas sementes de goiaba serrana os seguintes fungos: Aspergillus niger, Aspergillus flavus, Penicillium sp., Colletotrichum gloeosporioides, Fusarium sp., Curvularia sp., Alternaria sp., Trichoderma sp., Epicoccum sp. e Phomopsis sp. Os meios de cultura de BDA e V8 mostraram maior diversidade de colônias fúngicas, apresentando muitas vezes maior incidência quando comparados com o meio Blotter Test (Tabela 2).

Aspergillus niger foi encontrado em todas as amostras analisadas e em ambos os tratamentos (com e sem assepsia). A maior incidência foi na amostra LG, apresentando $11,7 \%$ de infestação. O lote CDIA (Concórdia, SC) apresentou a menor incidência média (1\%). O meio mais sensível na detecção de A. niger sem assepsia das sementes foi BDA. A adoção da assepsia superficial reduziu a incidência média de $A$. niger em seis procedências analisadas (Tabela 2).

A desinfestação das sementes para a realização do plaqueamento em BDA e V8 é importante, pois, na ausência dela os fungos que estão infestando as sementes podem atrapalhar a avaliação, sendo necessário, muitas vezes, repetir alguns testes, pela alta incidência de fungos. Isso ocorre porque esses meios de cultura são ricos em nutrientes, o que não ocorre no método Blotter Test (Lazarotto et al., 2010).

Aspergillus flavus apresentou maior incidência média para os lotes LG e menor para o lote CDIA, para ambos os tratamentos adotados, e o meio V8 foi o mais sensível na detecção desse fungo. Santos et al. (1998), avaliando a qualidade sanitária e fisiológica de sementes de Jacaranda cuspidifolia, observaram maior incidência de Aspergillus niger (19\%) e A. flavus (30\%) no método de plaqueamento em BDA em relação à incubação em Blotter Test, as quais foram de 9,5\% e 1,5\%, respectivamente. Resultado semelhante foi encontrado por Santos et al. (2001) em sementes de Acacia mearnsii.

O lote LG se destacou, apresentando 34\% de incidência média de Penicillium sp., sendo reduzida essa incidência em mais de $50 \%$ quando a assepsia superficial foi adotada. O método Botter Test foi o que permitiu uma detecção numericamente superior quando a assepsia foi adotada, não diferindo estatisticamente dos demais. Resultado semelhante foi encontrado por Schultz et al. (2003), que constataram superioridade 
do método Blotter Test em relação ao meio BDA para determinação da microbiota presente em sementes de pau-cigarra (Senna multijuga). Oliveira et al. (2012) verificou que Penicillium sp. manifestou-se de modo semelhante em tratamentos sem e com assepsia (álcool 70\%) em sementes de Schizolobium amazonicum.

Tabela 2. Incidência de fungos em sementes de goiaba serrana oriundas de municípios dos estados do Rio Grande do Sul e Santa Catarina, com e sem assepsia, submetidas ao teste de sanidade em meio de cultura agarizado e Blotter Test, em Lages, 2013.

Table 2. Incidence of fungi on guava feijoa seeds from municipalities in the States of Rio Grande do Sul and Santa Catarina, with and without asepsis, subjected to the test of sanity in the midst of agarizado culture and "Blotter Test". Lages, 2013.

\begin{tabular}{|c|c|c|c|c|c|c|c|c|c|}
\hline \multirow{3}{*}{ Fungo } & \multirow{3}{*}{$\begin{array}{l}\text { Proc. } \\
\text { sem. }\end{array}$} & \multicolumn{8}{|c|}{ Incidência (\%) } \\
\hline & & \multicolumn{4}{|c|}{ Sem assepsia } & \multicolumn{4}{|c|}{ Com assepsia } \\
\hline & & V8 & BDA & $\begin{array}{c}\text { Blotter } \\
\text { Test }\end{array}$ & Média & V8 & BDA & $\begin{array}{c}\text { Blotter } \\
\text { Test }\end{array}$ & Média \\
\hline \multirow{7}{*}{ 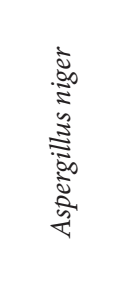 } & AT & $5 \mathrm{Aa}^{*}$ & $16 \mathrm{Aa}$ & $2 \mathrm{Aa}$ & $7,7 \mathrm{ab}$ & $13 \mathrm{Bc}$ & $0 \mathrm{Aa}$ & $4 \mathrm{Aba}$ & 5,7 a \\
\hline & CS & $5 \mathrm{Aa}$ & $11 \mathrm{Aa}$ & $1 \mathrm{Aa}$ & $5,7 \mathrm{ab}$ & $3 \mathrm{Aab}$ & $4 \mathrm{Aa}$ & $2 \mathrm{Aa}$ & 3,0 a \\
\hline & CMQ & $2 \mathrm{Aa}$ & $10 \mathrm{Aa}$ & $0 \mathrm{Aa}$ & $4,0 \mathrm{ab}$ & $4 \mathrm{Aab}$ & $7 \mathrm{Aa}$ & $2 \mathrm{Aa}$ & $4,3 \mathrm{a}$ \\
\hline & CDIA & $0 \mathrm{Aa}$ & $0 \mathrm{Aa}$ & $3 \mathrm{Aa}$ & $1,0 \mathrm{a}$ & $0 \mathrm{Aa}$ & $0 \mathrm{Aa}$ & $10 \mathrm{Ba}$ & $3,3 \mathrm{a}$ \\
\hline & LG & $13 \mathrm{Aa}$ & $9 \mathrm{Aa}$ & $13 \mathrm{Aa}$ & $11,7 \mathrm{~b}$ & $5 \mathrm{Aab}$ & $9 \mathrm{Aa}$ & $3 \mathrm{Aa}$ & $5,7 \mathrm{a}$ \\
\hline & SJ & $6 \mathrm{Aa}$ & $3 \mathrm{Aa}$ & $7 \mathrm{Aa}$ & $5,3 \mathrm{ab}$ & $0 \mathrm{Aa}$ & $1 \mathrm{Aa}$ & $4 \mathrm{Aa}$ & $1,7 \mathrm{a}$ \\
\hline & VA & $9 \mathrm{Aa}$ & $13 \mathrm{Aa}$ & $7 \mathrm{Aa}$ & $9,7 \mathrm{ab}$ & $6 \mathrm{Aab}$ & $0 \mathrm{Aa}$ & $1 \mathrm{Aa}$ & $2,3 a$ \\
\hline \multicolumn{2}{|l|}{ Média } & $5,7 \mathrm{~A}$ & $8,8 \mathrm{~A}$ & $4,7 \mathrm{~A}$ & & $4,4 \mathrm{~A}$ & $3,0 \mathrm{~A}$ & $3,7 \mathrm{~A}$ & \\
\hline \multicolumn{2}{|c|}{ CV (\%): } & \multicolumn{4}{|c|}{62,32} & \multicolumn{4}{|c|}{59,29} \\
\hline \multirow{7}{*}{ 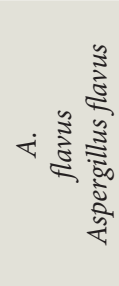 } & AT & $1 \mathrm{Aab}$ & $3 \mathrm{Aa}$ & $1 \mathrm{Aa}$ & $1,6 \mathrm{ab}$ & $9 \mathrm{Aab}$ & $3 \mathrm{Aa}$ & $5 \mathrm{Aa}$ & 5,7 a \\
\hline & CS & $19 \mathrm{Bc}$ & $5 \mathrm{Aa}$ & $15 \mathrm{Aba}$ & $13,0 \mathrm{c}$ & 9 Aabc & $5 \mathrm{Aa}$ & $5 \mathrm{Aa}$ & $6,3 \mathrm{ab}$ \\
\hline & CMQ & $7 \mathrm{Aabc}$ & $4 \mathrm{Aa}$ & $4 \mathrm{Aa}$ & $5,0 \mathrm{abc}$ & $12 \mathrm{Ab}$ & $3 \mathrm{Aa}$ & $11 \mathrm{Aa}$ & $8,7 \mathrm{ab}$ \\
\hline & CDIA & $0 \mathrm{Aa}$ & $0 \mathrm{Aa}$ & $1 \mathrm{Aa}$ & 0,3 a & $0 \mathrm{Aa}$ & $0 \mathrm{Aa}$ & $7 \mathrm{Aab}$ & $2,3 \mathrm{a}$ \\
\hline & LG & $13 \mathrm{Abc}$ & $8 \mathrm{Aa}$ & $3 \mathrm{Aa}$ & $8,0 \mathrm{bc}$ & $27 \mathrm{Bc}$ & $10 \mathrm{Aa}$ & $8 \mathrm{Aab}$ & $15,0 \mathrm{c}$ \\
\hline & SJ & 7 Aabc & $3 \mathrm{Aa}$ & $4 \mathrm{Aa}$ & $4,7 \mathrm{abc}$ & $0 \mathrm{Aa}$ & $1 \mathrm{Aa}$ & $6 \mathrm{Aa}$ & $2,3 \mathrm{a}$ \\
\hline & VA & 6 Aabc & $3 \mathrm{Aa}$ & $1 \mathrm{Aa}$ & $4,0 \mathrm{ab}$ & $17 \mathrm{Bbc}$ & $0 \mathrm{Aa}$ & $7 \mathrm{Aba}$ & $8,0 \mathrm{ab}$ \\
\hline \multicolumn{2}{|c|}{ Média } & 7,5 A & $4,0 \mathrm{~A}$ & $4,1 \mathrm{~A}$ & & $10,5 \mathrm{~B}$ & $3,1 \mathrm{~A}$ & $7,0 \mathrm{~B}$ & \\
\hline \multicolumn{2}{|c|}{ CV (\%): } & \multicolumn{4}{|c|}{55,82} & \multicolumn{4}{|c|}{46,4} \\
\hline \multirow{7}{*}{ 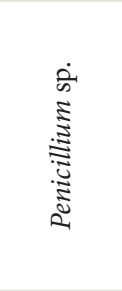 } & AT & $20 \mathrm{Aa}$ & $16 \mathrm{Aab}$ & $16 \mathrm{Aa}$ & 17,3 a & $25 \mathrm{Bb}$ & $6 \mathrm{Aa}$ & $4 \mathrm{Aa}$ & 11,7 a \\
\hline & CS & $14 \mathrm{Aa}$ & $21 \mathrm{Aab}$ & $12 \mathrm{Aa}$ & 15,7 a & $8 \mathrm{Aab}$ & $0 \mathrm{Aa}$ & $9 \mathrm{Aa}$ & 5,7 a \\
\hline & CMQ & $10 \mathrm{Aa}$ & $29 \mathrm{Aab}$ & $7 \mathrm{Aa}$ & 15,3 a & $6 \mathrm{Aab}$ & $17 \mathrm{Aa}$ & $8 \mathrm{Aa}$ & $10,3 \mathrm{a}$ \\
\hline & CDIA & $7 \mathrm{Aa}$ & $3 \mathrm{Aa}$ & $3 \mathrm{Aa}$ & 4,3 a & $0 \mathrm{Aa}$ & $2 \mathrm{Aa}$ & $8 \mathrm{Aa}$ & $3,3 \mathrm{a}$ \\
\hline & LG & $30 \mathrm{Aa}$ & $50 \mathrm{Bb}$ & $22 \mathrm{Aa}$ & $34,0 \mathrm{~b}$ & $1 \mathrm{Aa}$ & $17 \mathrm{Ba}$ & $19 \mathrm{Ba}$ & 13,3 a \\
\hline & SJ & $13 \mathrm{Aa}$ & $20 \mathrm{Aab}$ & $14 \mathrm{Aa}$ & $15,6 \mathrm{a}$ & $2 \mathrm{Aa}$ & $0 \mathrm{Aa}$ & $8 \mathrm{Aa}$ & $3,3 \mathrm{a}$ \\
\hline & VA & $12 \mathrm{Aa}$ & $21 \mathrm{Aa}$ & $13 \mathrm{Aa}$ & 15,3 a & $10 \mathrm{Aab}$ & $6 \mathrm{Aa}$ & $4 \mathrm{Aa}$ & $9,3 \mathrm{a}$ \\
\hline \multicolumn{2}{|c|}{ Média } & $16,4 \mathrm{~A}$ & $21,5 \mathrm{~B}$ & $12,4 \mathrm{~A}$ & & $7,7 \mathrm{~A}$ & $6,8 \mathrm{~A}$ & $9,4 \mathrm{~A}$ & \\
\hline \multicolumn{2}{|c|}{ CV (\%): } & \multicolumn{4}{|c|}{44,84} & \multicolumn{4}{|c|}{52,69} \\
\hline \multirow{7}{*}{ 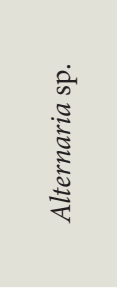 } & AT & $17 \mathrm{Aa}$ & $4 \mathrm{Aa}$ & $10 \mathrm{Aa}$ & $10,3 \mathrm{ab}$ & $0 \mathrm{Aa}$ & $6 \mathrm{Aa}$ & $5 \mathrm{Aa}$ & $3,7 \mathrm{abc}$ \\
\hline & CS & $10 \mathrm{Aa}$ & $1 \mathrm{Aa}$ & $2 \mathrm{Aa}$ & $4,3 \mathrm{a}$ & $6 \mathrm{Aab}$ & $3 \mathrm{Aa}$ & $3 \mathrm{Aa}$ & $4,0 \mathrm{abc}$ \\
\hline & CMQ & $14 \mathrm{Aa}$ & $12 \mathrm{Aa}$ & $7 \mathrm{Aa}$ & $11,0 \mathrm{ab}$ & $11 \mathrm{Bb}$ & $3 \mathrm{Aa}$ & $7 \mathrm{ABa}$ & $7,0 \mathrm{bc}$ \\
\hline & CDIA & $29 \mathrm{Aa}$ & $12 \mathrm{Aa}$ & $21 \mathrm{Aa}$ & $20,6 \mathrm{~b}$ & $14 \mathrm{Bb}$ & $3 \mathrm{Aa}$ & $7 \mathrm{ABa}$ & $8,0 \mathrm{c}$ \\
\hline & LG & $9 \mathrm{Aa}$ & $7 \mathrm{Aa}$ & $10 \mathrm{Aa}$ & $8,7 \mathrm{ab}$ & $0 \mathrm{Aa}$ & $3 \mathrm{Aa}$ & $0 \mathrm{Aa}$ & $1,0 \mathrm{a}$ \\
\hline & SJ & $6 \mathrm{Aa}$ & $4 \mathrm{Aa}$ & $3 \mathrm{Aa}$ & 4,3 a & $0 \mathrm{Aa}$ & $3 \mathrm{Aa}$ & $1 \mathrm{Aa}$ & $1,3 \mathrm{ab}$ \\
\hline & VA & $20 \mathrm{Aa}$ & $19 \mathrm{Aa}$ & $12 \mathrm{Aa}$ & $17,0 \mathrm{ab}$ & $12 \mathrm{Ab}$ & $7 \mathrm{Aa}$ & $9 \mathrm{Aa}$ & $9,3 \mathrm{c}$ \\
\hline \multicolumn{2}{|l|}{ Média } & $15 \mathrm{~A}$ & $8,4 \mathrm{~A}$ & 9,3 A & & $6,1 \mathrm{~A}$ & $4,0 \mathrm{~A}$ & $4,6 \mathrm{~A}$ & \\
\hline \multicolumn{2}{|l|}{ CV (\%): } & \multicolumn{4}{|c|}{51,73} & \multicolumn{4}{|c|}{48,92} \\
\hline
\end{tabular}

Proc. sem.: Procedência das sementes; ${ }^{*}$ Médias seguidas por mesma letra maiúscula na linha e minúscula na coluna não diferem entre si pelo teste de Tukey ao nível de 5\% de probabilidade; AT: Arroio do Tigre, RS; CS: Cachoeira do Sul, RS; CMQ: Camaquã, RS; CDIA: Concórdia, SC; LG: Lages, SC; SJ: São Joaquim, SC; VA: Venâncio Aires, RS. 
Tabela 2. Continuação...

Table 2. Continue...

\begin{tabular}{|c|c|c|c|c|c|c|c|c|c|}
\hline \multirow{3}{*}{ Fungo } & \multirow{3}{*}{$\begin{array}{l}\text { Proc. } \\
\text { sem. }\end{array}$} & \multicolumn{8}{|c|}{ Incidência (\%) } \\
\hline & & \multicolumn{4}{|c|}{ Sem assepsia } & \multicolumn{4}{|c|}{ Com assepsia } \\
\hline & & V8 & BDA & $\begin{array}{c}\text { Blotter } \\
\text { Test }\end{array}$ & Média & V8 & BDA & $\begin{array}{c}\text { Blotter } \\
\text { Test }\end{array}$ & Média \\
\hline \multirow[b]{8}{*}{ Média } & AT & $0 \mathrm{Aa}$ & $0 \mathrm{Aa}$ & $0 \mathrm{Aa}$ & $0,0 \mathrm{a}$ & $0 \mathrm{Aa}$ & $5 \mathrm{Aa}$ & $0 \mathrm{Aa}$ & $1,7 \mathrm{ab}$ \\
\hline & CS & $10 \mathrm{Bb}$ & $0 \mathrm{Aa}$ & $3 \mathrm{Aa}$ & $4,3 \mathrm{a}$ & $0 \mathrm{Aa}$ & $0 \mathrm{Aa}$ & $0 \mathrm{Aa}$ & $0,0 \mathrm{a}$ \\
\hline & CMQ & $0 \mathrm{Aa}$ & $0 \mathrm{Aa}$ & $0 \mathrm{Aa}$ & $0,0 \mathrm{a}$ & $0 \mathrm{Aa}$ & $0 \mathrm{Aa}$ & $0 \mathrm{Aa}$ & $0,0 \mathrm{a}$ \\
\hline & CDIA & $0 \mathrm{Aa}$ & $7 \mathrm{Bb}$ & $1 \mathrm{Aa}$ & 2,7 a & $1 \mathrm{Aa}$ & $5 \mathrm{Aa}$ & $4 \mathrm{Aa}$ & $3,3 \mathrm{ab}$ \\
\hline & LG & $18 \mathrm{Bb}$ & $9 \mathrm{Bb}$ & $11 \mathrm{Abb}$ & $12,7 \mathrm{~b}$ & $7 \mathrm{Aa}$ & $1 \mathrm{Aa}$ & $5 \mathrm{Aa}$ & $4,3 \mathrm{~b}$ \\
\hline & SJ & $0 \mathrm{Aa}$ & $5 \mathrm{Aab}$ & $0 \mathrm{Aa}$ & 1,7 a & $0 \mathrm{Aa}$ & $0 \mathrm{Aa}$ & $0 \mathrm{Aa}$ & 0,0 a \\
\hline & VA & $0 \mathrm{Aa}$ & $2 \mathrm{Aab}$ & $0 \mathrm{Aa}$ & $0,7 \mathrm{a}$ & $0 \mathrm{Aa}$ & $0 \mathrm{Aa}$ & $0 \mathrm{Aa}$ & 0,0 a \\
\hline & & $4,0 \mathrm{~A}$ & $3,3 \mathrm{~A}$ & $2,1 \mathrm{~A}$ & & $1,1 \mathrm{~A}$ & $1,6 \mathrm{~A}$ & $1,3 \mathrm{~A}$ & \\
\hline \multicolumn{2}{|l|}{ CV (\%): } & \multicolumn{4}{|c|}{42,09} & \multicolumn{4}{|c|}{59,22} \\
\hline \multirow{7}{*}{$\begin{array}{l}\dot{2} \\
\frac{n}{\tilde{n}} \\
\frac{2}{2} \\
\frac{1}{5} \\
\frac{2}{2}\end{array}$} & AT & $19 \mathrm{Bc}$ & $5 \mathrm{Aab}$ & $8 \mathrm{ABa}$ & $10,6 b$ & $0 \mathrm{Aa}$ & $7 \mathrm{Ba}$ & $4 \mathrm{Aa}$ & $3,7 \mathrm{a}$ \\
\hline & CS & $0 \mathrm{Aa}$ & $3 \mathrm{Aab}$ & $0 \mathrm{Aa}$ & $1,0 \mathrm{a}$ & $0 \mathrm{Aa}$ & $16 \mathrm{Ba}$ & $0 \mathrm{Aa}$ & $5,3 \mathrm{a}$ \\
\hline & CMQ & $12 \mathrm{Bbc}$ & $0 \mathrm{Aa}$ & $4 \mathrm{Aba}$ & $5,3 \mathrm{ab}$ & $0 \mathrm{Aa}$ & $5 \mathrm{Aa}$ & $0 \mathrm{Aa}$ & $1,7 \mathrm{a}$ \\
\hline & CDIA & $2 \mathrm{Aa}$ & $9 \mathrm{Aab}$ & $1 \mathrm{Aa}$ & $4,0 \mathrm{a}$ & $5 \mathrm{Aa}$ & $4 \mathrm{Aa}$ & $4 \mathrm{Aa}$ & $4,3 \mathrm{a}$ \\
\hline & LG & $5 \mathrm{Aab}$ & $0 \mathrm{Aa}$ & $0 \mathrm{Aa}$ & $1,7 \mathrm{a}$ & $3 \mathrm{Aa}$ & $0 \mathrm{Aa}$ & $0 \mathrm{Aa}$ & $1,0 \mathrm{a}$ \\
\hline & SJ & $0 \mathrm{Aa}$ & $6 \mathrm{Aab}$ & $1 \mathrm{Aa}$ & $2,3 \mathrm{a}$ & $0 \mathrm{Aa}$ & $0 \mathrm{Aa}$ & $0 \mathrm{Aa}$ & 0,0 a \\
\hline & VA & $8 \mathrm{Aabc}$ & $10 \mathrm{Ab}$ & $3 \mathrm{Aa}$ & $7,0 \mathrm{ab}$ & $0 \mathrm{Aa}$ & $12 \mathrm{Ba}$ & $0 \mathrm{Aa}$ & $4,0 \mathrm{a}$ \\
\hline \multicolumn{2}{|l|}{ Média } & $6,5 \mathrm{~B}$ & $4,7 \mathrm{AB}$ & $2,4 \mathrm{~A}$ & & $1,1 \mathrm{~A}$ & $6,2 \mathrm{~B}$ & $1,1 \mathrm{~A}$ & \\
\hline \multicolumn{2}{|l|}{ CV (\%): } & \multicolumn{4}{|c|}{47,7} & \multicolumn{4}{|c|}{70,4} \\
\hline \multirow{7}{*}{ 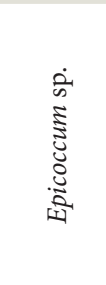 } & AT & $0 \mathrm{Aa}$ & $3 \mathrm{Aab}$ & $1 \mathrm{Aa}$ & $1,3 \mathrm{a}$ & $0 \mathrm{Aa}$ & $0 \mathrm{Aa}$ & $9 \mathrm{Ba}$ & 3,0 a \\
\hline & CS & $0 \mathrm{Aa}$ & $6 \mathrm{Aab}$ & $4 \mathrm{Aab}$ & $3,3 \mathrm{a}$ & $0 \mathrm{Aa}$ & $0 \mathrm{Aa}$ & $5 \mathrm{Ba}$ & $1,7 \mathbf{a}$ \\
\hline & CMQ & $0 \mathrm{Aa}$ & $10 \mathrm{Bb}$ & $0 \mathrm{Aa}$ & $3,3 \mathrm{a}$ & $0 \mathrm{Aa}$ & $0 \mathrm{Aa}$ & $6 \mathrm{Ba}$ & $2,0 \mathrm{a}$ \\
\hline & CDIA & $5 \mathrm{Aab}$ & $3 \mathrm{Aab}$ & $3 \mathrm{Aab}$ & $3,7 \mathrm{a}$ & $3 \mathrm{Aa}$ & $3 \mathrm{Aa}$ & $1 \mathrm{Aa}$ & $2,3 \mathrm{a}$ \\
\hline & LG & $14 \mathrm{Ab}$ & $10 \mathrm{Aab}$ & $13 \mathrm{Ab}$ & $12,3 \mathrm{~b}$ & $1 \mathrm{Aa}$ & $0 \mathrm{Aa}$ & $1 \mathrm{Aa}$ & $0,7 \mathbf{a}$ \\
\hline & SJ & $2 \mathrm{Aab}$ & $0 \mathrm{Aa}$ & $0 \mathrm{Aa}$ & $0,7 \mathrm{a}$ & $0 \mathrm{Aa}$ & $1 \mathrm{Aa}$ & $1 \mathrm{Aa}$ & $0,7 a$ \\
\hline & VA & $0 \mathrm{Aa}$ & $4 \mathrm{Aab}$ & $0 \mathrm{Aa}$ & $1,3 \mathrm{a}$ & $0 \mathrm{Aa}$ & $0 \mathrm{Aa}$ & $1 \mathrm{Aa}$ & $0,3 \mathrm{a}$ \\
\hline \multicolumn{2}{|l|}{ Média } & $3,0 \mathrm{~A}$ & $3.0 \mathrm{~A}$ & $5,1 \mathrm{~A}$ & & $0,6 \mathrm{~A}$ & $0,6 \mathrm{~A}$ & $3,4 \mathrm{~B}$ & \\
\hline \multicolumn{2}{|c|}{$\mathrm{CV}(\%)$} & & 51,67 & & & \multicolumn{4}{|c|}{50,02} \\
\hline \multirow{7}{*}{ 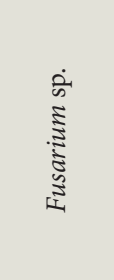 } & AT & $0 \mathrm{Aa}$ & $7 \mathrm{Aab}$ & $0 \mathrm{Aa}$ & $2,3 \mathrm{ab}$ & $0 \mathrm{Aa}$ & $0 \mathrm{Aa}$ & $5 \mathrm{Aab}$ & $1,7 \mathbf{a}$ \\
\hline & CS & $6 \mathrm{Aab}$ & $16 \mathrm{Ab}$ & $3 \mathrm{Aa}$ & $8,3 \mathrm{ab}$ & $0 \mathrm{Aa}$ & $2 \mathrm{Aa}$ & $1 \mathrm{Aa}$ & $1,0 \mathrm{a}$ \\
\hline & CMQ & $9 \mathrm{ABab}$ & $16 \mathrm{Bb}$ & $1 \mathrm{Aa}$ & $8,7 \mathrm{ab}$ & $0 \mathrm{Aa}$ & $1 \mathrm{Aa}$ & $0 \mathrm{Aa}$ & $0,3 a$ \\
\hline & CDIA & $13 \mathrm{Ab}$ & $4 \mathrm{Aab}$ & $7 \mathrm{Aa}$ & $8,0 \mathrm{ab}$ & $15 \mathrm{Ab}$ & $10 \mathrm{Aa}$ & $11 \mathrm{Ab}$ & $12,0 \mathrm{~b}$ \\
\hline & LG & $14 \mathrm{Ab}$ & $14 \mathrm{Aab}$ & $6 \mathrm{Aa}$ & $11,33 \mathrm{~b}$ & $0 \mathrm{Aa}$ & $2 \mathrm{Aa}$ & $0 \mathrm{Aa}$ & $0,7 \mathbf{a}$ \\
\hline & SJ & $8 \mathrm{Aab}$ & $7 \mathrm{Aab}$ & $6 \mathrm{Aa}$ & $7,0 \mathrm{ab}$ & $6 \mathrm{Aab}$ & $1 \mathrm{Aa}$ & $4 \mathrm{Aab}$ & $3,7 \mathbf{a}$ \\
\hline & VA & $8 \mathrm{Aab}$ & $0 \mathrm{Aa}$ & $2 \mathrm{Aa}$ & $2,0 \mathrm{a}$ & $8 \mathrm{Bab}$ & $0 \mathrm{Aa}$ & $2 \mathrm{Abab}$ & 3,3 a \\
\hline \multicolumn{2}{|l|}{ Média } & $7,7 \mathrm{AB}$ & 9,14 B & $3,5 \mathrm{~A}$ & & $4,1 \mathrm{~A}$ & $2,3 \mathrm{~A}$ & $3,3 \mathrm{~A}$ & \\
\hline CV (\%) & & & 52,2 & & & & & & \\
\hline & AT & $22 \mathrm{Bbc}$ & $8 \mathrm{Aa}$ & $8 \mathrm{Aa}$ & $12,7 \mathrm{bc}$ & $14 \mathrm{Bb}$ & $7 \mathrm{ABa}$ & $4 \mathrm{Aab}$ & $8,3 \mathrm{c}$ \\
\hline के & CS & $35 \mathrm{Bc}$ & $9 \mathrm{Aa}$ & $10 \mathrm{Aa}$ & $18,0 \mathrm{c}$ & $9 \mathrm{Bab}$ & $8 \mathrm{ABa}$ & $0 \mathrm{Aa}$ & $5,7 \mathrm{abc}$ \\
\hline ఏ & CMQ & $2 \mathrm{Aab}$ & $0 \mathrm{Aa}$ & $0 \mathrm{Aa}$ & $0,7 \mathbf{a}$ & $0 \mathrm{Aa}$ & $4 \mathrm{Aa}$ & $0 \mathrm{Aa}$ & $1,3 \mathrm{ab}$ \\
\hline$\frac{\bar{z}}{0}$ & CDIA & $1 \mathrm{Aa}$ & $9 \mathrm{Aa}$ & $0 \mathrm{Aa}$ & $3,3 \mathrm{ab}$ & $0 \mathrm{Aa}$ & $0 \mathrm{Aa}$ & $0 \mathrm{Aa}$ & $0,0 a$ \\
\hline : & LG & $0 \mathrm{Aa}$ & $0 \mathrm{Aa}$ & $0 \mathrm{Aa}$ & $0,0 \mathrm{a}$ & $11 \mathrm{Bb}$ & $0 \mathrm{Aa}$ & $12 \mathrm{Bb}$ & $7,7 \mathrm{bc}$ \\
\hline E & SJ & $8 \mathrm{Aab}$ & $14 \mathrm{Aa}$ & $3 \mathrm{Aa}$ & 8,3 abc & $0 \mathrm{Aa}$ & $2 \mathrm{Aa}$ & $0 \mathrm{Aa}$ & $0,7 \mathrm{a}$ \\
\hline & VA & $3 \mathrm{Aab}$ & $0 \mathrm{Aa}$ & $0 \mathrm{Aa}$ & $1,0 \mathrm{a}$ & $0 \mathrm{Aa}$ & $0 \mathrm{Aa}$ & $0 \mathrm{Aa}$ & 0,0 a \\
\hline Média & & 10,1 B & $5,7 \mathrm{AB}$ & $3,0 \mathrm{~A}$ & & $4,8 \mathrm{~A}$ & $5,8 \mathrm{~A}$ & $2,3 \mathrm{~A}$ & \\
\hline CV (\%) & & & 65,62 & & & & & & \\
\hline
\end{tabular}

Proc. sem.: Procedência das sementes; ${ }^{*}$ Médias seguidas por mesma letra maiúscula na linha e minúscula na coluna não diferem entre si pelo teste de Tukey ao nível de 5\% de probabilidade; AT: Arroio do Tigre, RS; CS: Cachoeira do Sul, RS; CMQ: Camaquã, RS; CDIA: Concórdia, SC; LG: Lages, SC; SJ: São Joaquim, SC; VA: Venâncio Aires, RS. 
O meio de cultura BDA apresentou incidência média superior de Penicillum sp. somente quando a assepsia não foi adotada. Rego (2005) considera que o método Blotter Test apresenta limitações para a detecção de fungos de crescimento lento e localizados internamente. A superioridade do meio BDA deve-se, em grande parte, ao fato de se tratar de um substrato rico em nutrientes (Magalhães et al., 2008), característica que pode favorecer o desenvolvimento dos fungos em maior intensidade e velocidade.

Parisi (2012) e Oliveira (2011) também relataram incidência de Aspergillus sp. e Penicillium sp. em sementes de Inga vera e Eugenia spp. Esses fungos também apresentaram elevada incidência em sementes de Zollernia ilicifolia Vog. (pau-santo), Plathymenia reticulata Benth. (vinhático do campo), Cassia sp. (canafístula) e Handroanthus sp. (ipê), prejudicando a qualidade das sementes com a queda de sua viabilidade (Carneiro, 1990).

Bajpai \& Patil (1997) e Pinto (2003) destacam que as espécies dos gêneros Aspergillus e Penicillium têm a capacidade de produzir enzimas extracelulares sobre diversos substratos, destacando-se as lipases, proteases, amilases, pectinases, tanases e lacases.

Epicoccum sp. teve incidência média reduzida se comparada à dos demais infestantes, novamente o lote LG se destaca com ocorrência superior desse fungo, para ambos os tratamentos. O método Blotter Test permitiu maior desenvolvimento de Epicoccum sp., diferindo estatisticamente das demais formas de detecção apenas quando a assepsia foi adotada, resultado semelhante foi encontrado por Maciel et al. (2012) em sementes de Parapiptadenia rigida.

Botelho et al. (2008) detectaram A. flavus, A. niger, Penicillium sp. e Epicoccum sp. em sementes de Schinus terebinthifolius, enquanto que Santos et al. (1997) encontraram os mesmos fungos associados com sementes de Dipteryx alata.

Faiad et al. (2004) identificaram A. niger em sementes de goiaba serrana, constatando que a sua incidência aumentou quando se aumentava o período de armazenamento das sementes. Machado et al. (2004) relataram que tanto Aspergillus sp. como Penicillium sp. são fungos associados à deterioração de sementes em condições de armazenamento inadequado, mas a associação pode ocorrer logo após a colheita.
Alternaria sp. foi detectado em todas as amostras, sendo o lote CDIA o que apresentou maior incidência média $(20,6 \%)$ para o tratamento sem assepsia. O meio mais sensível para detecção de Alternaria sp. em sementes de goiaba serrana foi o V8, não apresentando diferença significativa em relação ao meio de cultura BDA e ao método Blotter Test. A assepsia reduziu a incidência de Alternaria sp. em todas as procedências analisadas. Resultados semelhantes foram encontrados por Santos et al. (2002), que identificaram Alternaria sp. em sementes de quatro espécies arbóreas da Mata Atlântica (Enterolobium contortisiliquum, Peltophorum dubium, Bauhinia forficata e Poecilanthe parviflora), que tiveram incidência reduzida quando adotada assepsia com álcool a 70\% (por 30 segundos) e, em seguida, com hipoclorito de sódio a 1\% (por 2 minutos), sendo posteriormente lavadas com água destilada esterilizada. O gênero Alternaria foi relatado em associação com 58 espécies florestais nativas do Cerrado brasileiro, causando descoloração das sementes, redução da taxa de germinação, baixo vigor de plântulas, necrose nas raízes e morte de plântulas em viveiro (Faiad et al., 2004).

O meio V8 foi mais sensível na detecção de Curvularia sp. quando as sementes não foram submetidas a desinfestação e o meio BDA, quando a assepsia das sementes foi adotada. O lote LG apresentou as maiores médias de incidência para o tratamento sem e com assepsia ( $12,7 \%$ e $4,3 \%$, respectivamente). Para os lotes CS, SJ e VA, a assepsia superficial mostrou-se eficiente, erradicando Curvularia sp. e mostrando que esse fungo estava associado aos tecidos externos da semente. O meio de cultura V8 foi mais sensível para a sua detecção quando não se adota assepsia e o meio de cultura de BDA, quando a assepsia é adotada.

Phomopsis sp. foi detectado nas sete procedências, e o meio V8 apresentou-se como o mais sensível na sua detecção, havendo diferença significativa entre as formas de detecção para o tratamento sem assepsia. A desinfestação das sementes mostrou-se eficiente, reduzindo as médias gerais de incidência e erradicando Phomopsis sp. na procedência SJ.

Ruiz et al. (2004) relataram que Phomopsis sp. foi o fungo mais frequentemente encontrado em sementes de Cedrela fissilis. Não se tem relatos de que Phomopsis sp. seja patogênico para goiaba serrana, porém Santos et al. (1997), inoculando Phomopsis sp. em sementes de baru (Dipterips alata), comprovaram redução na germinação 
e presença de plântulas sintomáticas. Anjos et al. (2001) relataram que Phomopsis sp. causou queima das folhas em aroeira (Myracrodruon urundeuva). Cherobini et al. (2004) constataram 9,5\% de incidência média de Phomopsis sp. em sementes de goiaba serrana utilizando papel como substrato.

O lote LG apresentou a maior incidência média de Fusarium spp. (11,3\%) sem desinfestação, sendo ele detectado em todas as procedências. Teve sua incidência aumentada no lote CDIA, passando de $8,0 \%$ para $12,0 \%$ quando a assepsia foi adotada, demonstrando, nesse caso, não ser eficiente a desinfestação e comprovando que as estruturas desse fungo estão localizadas na parte interna da semente. A alta incidência média de Fusarium spp. na procedência CDIA pode estar relacionada com o método de coleta dos frutos de goiaba serrana, já que alguns foram coletados direto do chão. Segundo Menten \& Bueno (1987), o inóculo externo pode vir do solo, mostrando, nesse caso, que parte do inóculo teria se originado de fonte externa. No presente trabalho foi comprovada a transmissão de Fusarium sp. de semente para plântula de goiaba serrana, ocasionando lesões em plântulas, principalmente nas raízes, que tiveram seu tamanho reduzido.

Algumas espécies do gênero Fusarium foram encontradas causando danos em plântulas do gênero Pinus, Stryphnodendron adstringens Mart. (barbatimão), Anadenanthera macrocarpa Benth. (angico vermelho),
Copaifera langsdorffii Desf. (copaíba) e Dalbergia nigra (Vell.) Benth. (jacarandá da Bahia) (Sales, 1992; Mesquita, 1999).

Trichoderma spp. foi encontrado associado às sementes de todas as procedências analisadas. No geral, a assepsia mostrou-se eficiente reduzindo a incidência de Trichoderma spp. em seis procedências, erradicando-a no lote CDIA. Os meios agarizados apresentaram-se mais sensíveis na sua detecção. Fungos do gênero Trichoderma estão entre os microrganismos mais estudados como agentes no biocontrole de fitopatógenos promotores da germinação de sementes e do crescimento vegetal (Altomare et al., 1999).

O meio V8 foi o mais sensível na detecção de C. gloeosporioides (Figura 1) para os dois tratamentos adotados. O patógeno foi encontrado associado às sementes das procedências CDIA e SJ.

Martins et al. (2005), testando C. gloeosporioides isolados da cultura do maracujá frente a ação dos meios líquidos V8 (Campbell $\left.{ }^{\circledR}\right), \mathrm{BD}$ (batata-dextrose) e $\mathrm{BD}+\mathrm{EL}$ (batata-dextrose + extrato de levedura), verificaram que V8 possui boa ação de esporulação.

Para a procedência SJ, esse patógeno teve incidência média de $18 \%$ e $5 \%$ (sem e com desinfestação, respectivamente) no meio V8; $27 \%$ e $3 \%$ (sem e com desinfestação, respectivamente) no meio $\mathrm{BDA}$; e
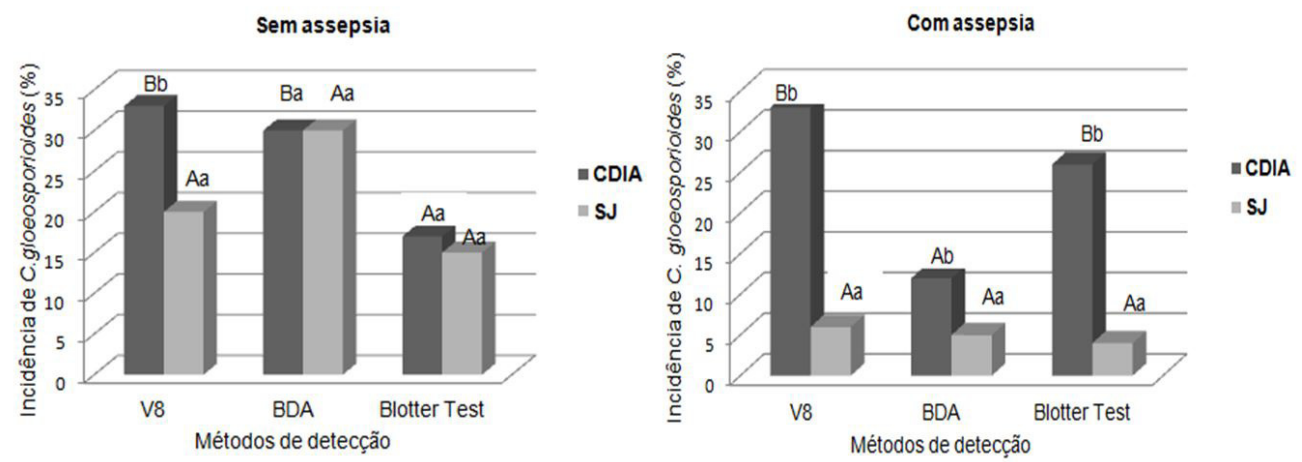

Figura 1. Incidência de Colletotrichum gloeosporioides em sementes de goiaba serrana obtidas nos municípios de Concórdia, SC (CDIA), e São Joaquim, SC (SJ), em meio de cultura de batata-dextrose-ágar, meio de cultura V8 e método Blotter Test, submetidas ou não à assepsia; as letras maiúsculas representam o teste de Tukey a $5 \%$ entre métodos de detecção para a mesma procedência, e as minúsculas, entre procedências.

Figure 1. Incidence of Colletotrichum gloeosporioides in seeds of guava feijoa obtained in the municipalities of Concord/SC (CDIA) and São Joaquim-SC (SJ), in the culture medium of Potato-Dextrose-Àgar, V8 culture medium and method "Blotter Test", subjected or not to asepsis. The uppercase letters represent the Tukey test to 5\% between detection methods for the same origin and the lowercase between provenances. 
$12 \%$ e $2 \%$ (sem e com desinfestação, respectivamente) no método Blotter Test.

A procedência CDIA apresentou as seguintes incidências médias do patógeno: 32\% e 30\% (sem e com desinfestação, respectivamente) para o meio V8-ágar; $27 \%$ e $12 \%$ (sem e com desinfestação, respectivamente) para o meio BDA; e 15\% e 25\% (sem e com desinfestação, respectivamente) no método Blotter Test.

Mendes et al. (2005) indentificou C. gloeosporioides em sementes de Mimosa caesalpiniaefolia, detectando maior incidência quando realizada a desinfestação, indicando maior quantidade de inóculo na parte interior da semente. No presente trabalho, a incidência média do patógeno foi maior quando a assepsia foi adotada, apenas na procedência CDIA, no método Blotter Test.

Hellwig \& Ueno (2009) relataram que C. gloeosporioides está associado à seca de ramos e à antracnose em frutos de goiaba serrana. A sua transmissão para plântula via semente foi comprovada no teste de transmissão de fungos, causando lesões escuras, necróticas, de tamanho e forma variáveis nos folíolos (Fantinel, 2014).

$\mathrm{O}$ período em que as sementes permaneceram armazenadas até a realização dos testes de sanidade, no presente trabalho, pode ter influenciado na incidência do fungo. Faiad et al. (2004), avaliando a sobrevivência de C. gloeosporioides em sementes de goiaba serrana durante o armazenamento a $8{ }^{\circ} \mathrm{C}$, por um período de seis meses, verificaram incidência média inicial de $87 \%$ e final de 67\%. Segundo Neergaard (1983), condições de armazenamento, que favorecem a longevidade das sementes, são geralmente as mesmas que favorecem a sobrevivência dos patógenos.

\section{CONCLUSÕES}

Os meios agarizados são mais sensíveis na detecção de fungos em sementes de goiaba serrana.

A assepsia das sementes com álcool 70\%, hipoclorito $1 \%$ e água destilada durante 30 segundos reduz a incidência de fungos infestantes em sementes de goiaba serrana, sendo indicada quando se realiza teste de sanidade com sementes dessa espécie.

\section{AGRADECIMENTOS}

À Universidade do Estado de Santa Catarina e aos professores Luciana Magda de Oliveira e Ricardo Trezzi Casa.

\section{STATUS DA SUBMISSÃO}

Recebido: 1 abr., 2014

Aceito: 26 jun., 2016

AUTOR(ES) PARA CORRESPONDÊNCIA

\section{Vinícius Spolaor Fantinel}

Departamento de Engenharia Florestal, Universidade do Estado de Santa Catarina UDESC, Av. Luiz de Camões, 2090, Bairro Conta Dinheiro, CEP 88520-000, Lages, SC, Brasil e-mail: vinispofan@hotmail.com

\section{REFERÊNCIAS}

Altomare C, Norvell WA, Bjorkman T, Harman GE. Solubilization of phosphates and micronutrients by the plant-growth-promoting and biocontrol fungus Trichoderma harzianum Rifai 1295-22. Applied and Environmental Microbiology 1999; 65(7): 2926-2933. PMid:10388685.

Amarante CVT. SANTOS KL. Goiabeira-serrana (Acca sellowiana). Revista Brasileira de Fruticultura [online]. 2011 [citado em 2014 abr 1]; 33(1): 001-334. Disponível em: http:// www.scielo.br/scielo.php?script=sci_arttext\&pid=S010029452011000100042\&lng=en\&nrm=iso

Anjos JRN, Charchar MJA, Guimarães DP. Ocorrência de queima das folhas causada por Phomopsis sp. em aroeira no Distrito Federal. Fitopatologia Brasileira 2001; 26(3): 549-650. http://dx.doi.org/10.1590/S010041582001000300012 .

Bajpai B, Patil S. Induction of tannin acyl hydrolases (EC 3.1.1.20) activity in some members of fungi imperfecti. Enzyme and Microbial Technology 1997; 20(8): 612-614. http://dx.doi.org/10.1016/S0141-0229(96)00206-2.

Barnett HL, Henter BB. Illustrated genera of imperfect fungi. 4th ed. Saint Paul: The American Phytopathological Society; 1998. p. 218.

Botelho LS, Moraes MHD, Menten JOM. Fungos associados às sementes de ipê-amarelo (Tabebuia serratifolia) e ipê-roxo (Tabebuia impetiginosa): incidência, efeito na germinação e transmissão para as plântulas. Summa Phytopathologica 2008; 34(4): 343-348. http://dx.doi. org/10.1590/S0100-54052008000400008. 
Brasil. Ministério da Agricultura, Pecuária e Abastecimento. Manual de análise sanitária de sementes. Brasília: Mapa; 2009. p. 200.

Carneiro JS. Qualidade sanitária de sementes de espécies florestais em Paraopeba, MG. Fitopatologia Brasileira 1990; 15(1): 75-77.

Cherobini EAI, Muniz MFB, Hoppe JM, Ávila AL, Camargo RF. Qualidade sanitária de sementes de Eugenia involucrata DC, Eugenia pyriformis cambessedes, Feijoa sellowiana Berg, Psidium cattleianum sabine. In: Palestras e Resumos do Simpósio Brasileiro de Patologia de Sementes; 2004; João Pessoa. João Pessoa: Embrapa Agroindústria Tropical; 2004. p. 163.

Coutinho WM, Machado JC, Vieira MGGC, Guimarães RM, Ferreira DF. Uso da restrição hídrica na embebição ou retardamento da germinação de sementes de arroz e feijão submetidas ao teste de sanidade em meio ágar-água. Revista Brasileira de Sementes 2001; 23(2): 127-135. http:// dx.doi.org/10.17801/0101-3122/rbs.v23n2p127-135.

Ducroquet JPHJ. Goiabeira-Serrana: fatores climáticos trazem a pesquisa de volta ao centro de origem da espécie. Agropecuária Catarinense 1996; 9(3): 13-15.

Faiad MGR, Ramos VR, Wetzel MMV. Patologia de sementes de espécies florestais do cerrado. In: Palestras e Resumos do VIII Simpósio Brasileiro de Patologia de Sementes; 2004; João Pessoa. Fortaleza: Embrapa Agroindústria Tropical, 2004. p. 171.

Fantinel VS. Fungos associados às sementes de goiabaserrana: detecção, efeitos na qualidade das sementes, transmissão para plântulas e controle [dissertação]. Lages: Universidade do Estado de Santa Catarina; 2014.

Fernandez MR. Manual para laboratorio de fitopatologia. Passo Fundo: Embrapa-CNTP; 1993. p. 128.

Ferreira DF. Análises estatísticas por meio do Sisvar para Windows versão 4.0. In: Anais da $45^{a}$ Reunião Anual da Região Brasileira da Sociedade Internacional de Biometria; 2000; São Carlos. São Carlos: SIB; 2000. p. 255-258.

Hellwig T, Ueno B. Levantamento de fitopatógenos causadores de doenças em frutíferas nativas na região sul do Rio Grande do Sul. Revista Brasileira de Agroecologia 2009; 4(2): 1560-1564.

Lazarotto M, Muniz MFB, Santos ÁF. Detection, transmission, pathogenicity and chemical treatment of fungi in Ceiba speciosa seeds. Summa Phytopathologica 2010; 36(2): 134-139. http://dx.doi.org/10.1590/S01005405201000020000 .

Lucca AO Fo. Metodologia dos testes de sanidade de sementes. In: Soave J, Wetzel MMVS, editores. Patologia de sementes. Campinas: Fundação Cargill; 1987.p. 276-298.

Machado AA, Muniz MFB, Hoppe JM, Camargo R. Influência de diferentes tratamentos se sementes de cedro (Cedrela fissilis Vell.) e cerejeira (Eugenia involucrata
DC.) sobre a incidência de fungos de armazenamento. Fitopatologia Brasileira 2004; 29(supl.): 354.

Maciel CG, Muniz MFB, Santos ÁF, Lazarotto M. Detecção, transmissão e patogenicidade de fungos em sementes de Parapiptadenia rigida (angico-vermelho). Summa Phytopathologica 2012; 38(4): 323-328. http://dx.doi. org/10.1590/S0100-54052012000400009.

Maddox DA. Implications of new technologies for seed health testing and the worldwide movement of seed. Seed Science Research 1998; 8(02): 277-284. http://dx.doi. org/10.1017/S0960258500004177.

Magalhães HM, Catão HCRM, Sales NLP, Lima NF, Lopes PSN. Qualidade sanitária de sementes de coquinho-azedo (Butia capitata) no Norte de Minas Gerais. Ciência Rural 2008; 38(8): 2371-2374. http://dx.doi.org/10.1590/S010384782008000800045 .

Martins I, Mello SCM, Ávila ZR, Pádua RR, Peixoto JR. Produção de Colletotrichum gloesporioides em meios líquidos. Brasília: EMBRAPA; 2005. p. 6. Circular Técnica n. 45.

Mendes SS, Santos PR, Santana GC, Ribeiro GT, Mesquita JB. Levantamento, patogenicidade e transmissão de fungos associados às sementes de sabiá (Mimosa caesalpiniaefolia Benth). Revista Ciência Agronômica 2005; 36(1): 118-122.

Menten JOM, Bueno JT. Transmissão de patógenos pelas sementes. In: Soave J, Wetzel MMVS, editores. Patologia de sementes. Campinas: Fundação Cargill; 1987. p. 164-191.

Mesquita JB. Patologia de sementes de angico-vermelho, copaíba e jacarandá-da-Bahia [tese]. Viçosa: Universidade Federal de Viçosa; 1999. 53 p.

Neergaard P. Seed pathology. London: The Macmillan Press; 1983. p. 839.

Oliveira CF. Conservação de sementes de Eugenia uniflora Lam. e Inga vera Penn.: qualidade sanitária e taxas respiratórias [dissertação]. São Paulo: Instituto de Botânica de São Paulo; 2011.

Oliveira JD, Silva JB, Dapont EC, Souza LMS, Ribeiro AAL. Métodos para detecção de fungos e assepsia de sementes de Schizoobium amazonicum (Caesalpinioideae). Bioscience Journal, 2012; 28(6): 945-953.

Parisi JJD. Associação entre fungos e a viabilidade de embriões de Inga vera [dissertação]. Campinas: Universidade Estadual de Campinas, Faculdade de Engenharia Agrícola; 2012.

Pinto GAS. Produção de Tanase por Aspergillus niger [tese]. Rio de Janeiro: Universidade Federal do Rio de Janeiro; 2003.

Rego AM. Análise sanitária na produção de sementes de grandes culturas. In: ZAMBOLIM L, editor. Sementes: qualidade fitossanitária. Viçosa: UFV; 2005. p. 267-294.

Reis EM, Almeida MF. Comparison of methods to detect leaf and head blighting fungi in small grain seeds. Summa Phytopathologica 1999; 25: 364-367. 
Reis EM, Casa RT. Doenças dos cereais de inverno: diagnose, epidemiologia e controle. 2. ed. Lages: Graphel; 2007.

Ruiz RRF, Santos AF, Medeiros ACS, Jaccoud DS Fo. Fungos associados às sementes de cedro. Summa Phytopathologica 2004; 30(4): 494-496.

Sales NL. Efeito da população fúngica e do tratamento e do tratamento químico no desempenho de sementes de ipê-amarelo, ipê-roxo e barbatimão [dissertação] Lavras: Escola Superior de Agricultura de Lavras; 1992.

Santos AF, Medeiros ACS, Santana DL. Fungos associados a sementes de espécies arbóreas da mata atlântica. Colombo: Embrapa/CNPF; 2002. p. 51-60. Boletim de Pesquisa Florestal n. 42.

Santos FEM, Sobrosa RC, Costa IFD, Corder MPM. Detecção de fungos patogênicos em sementes de acácianegra (Acacia mearnsii De Wild.). Ciência Florestal 2001; 11(1): 13-20.

Santos KL, Siminski A, Ducroquet JPHJ, Guerra MP, Peroni N, Nodari RO. Acca sellowiana. In: Coradin L, Siminski A, Reis A. Espécies nativas da flora brasileira de valor econômico atual ou potencial: plantas para o futuro - Região Sul. Brasília: MMA; 2011. p. 111-130.
Santos MF, Ribeiro WRC, Faiad MGR, Salomão AN. Avaliação da qualidade sanitária e fisiológica das sementes de caroba. Revista Brasileira de Sementes 1998; 20(1): 1-6.

Santos MF, Ribeiro WRC, Faiad MGR, Sano SM. Fungos associados às sementes de baru (Dipterys alata Vog.). Revista Brasileira de Sementes 1997; 19(1): 135-139.

Schultz VS, Santos AF, Medeiros ACS. Qualidade sanitária de sementes de pau-cigarra (Senna multijuga). Boletim de Pesquisa Florestal 2003; 47: 123-128.

Souza CV, Lorenzi H. Botânica sistemática: guia ilustrado para identificação das famílias de angiospermas da flora brasileira, baseado na APGII. Nova Odessa: Instituto Plantarum; 2005.

Sutton BC. The genus Glomerella and its anamorph. In: Bailey JA, Jeger MJ, editors. Colletotrichum: biolody, pathology and control. England: CAB International Wallingford; 1992. p. 1-26

Wrege MS, Steinmetz S, Reisser C Jr, Almeida IR, editors. Atlas climático da Região Sul do Brasil: Estados do Paraná, Santa Catarina e Rio Grande do Sul. Pelotas: Embrapa Clima Temperado; Colombo: Embrapa Florestas; 2011. p. 333. il. color. 